\title{
Sabah Traditional Chinese Medicine Database
}

\author{
Aslina Baharum ${ }^{1}$, Neoh Yee Jin ${ }^{2}$, Shaliza Hayati A. Wahab ${ }^{3}$, Mohd Helmy Abd Wahab ${ }^{4}$, \\ Radzi Ambar ${ }^{5}$, Nurul Hidayah Mat Zain ${ }^{6}$ \\ ${ }^{1,2,3}$ Faculty of Computing and Informatics, Universiti Malaysia Sabah, 88400 Kota Kinabalu, Sabah, Malaysia \\ ${ }^{4,5}$ Faculty of Electric and Electronic, Universiti Tun Hussein Onn Malaysia, 86400 Batu Pahat, Johor, Malaysia \\ ${ }^{6}$ Faculty of Computer and Mathematicla Sciences, Universiti Teknologi MARA, Jasin Campus, \\ 77300 Merlimau, Melaka, Malaysia
}

\begin{tabular}{l}
\hline \hline Article Info \\
\hline Article history: \\
Received May 04, 2018 \\
Revised Jul 20, 2018 \\
Accepted Aug 15, 2018 \\
\hline
\end{tabular}

Keywords:

Database

Medicine

Sabah

Traditional chinese

\begin{abstract}
As technology grows, people tend to use or apply anything digitalized instead of printed, especially for references. This is because the printed form references are not easy to find. Even if the references are found successfully, it has already cost a lot of time, money, energy, etc. At the same time, people also put more emphasize on health issues. They are beginning to be more alert in fields that they have ignored before, such as traditional medicine and Chinese medicine. Based on these two points, it can be said that the effort of transforming Traditional Chinese Medicine (TCM) from printed based reference into online reference as a database is a public beneficial effort. There are a lot of online TCM database outside of Malaysia, especially from the People's Republic of China, Hong Kong, and Taiwan. Those herbal remedies from overseas are somewhat different from the herbal remedies in Malaysia due to the habits and occurrences of the herbs. Through this project, it is hoped that this database will help the local people to discover and identify the herbs that they could find in the surrounding area. The objectives of this project are to identify the validity of the information of the Sabah TCM using mixed method, to develop the Sabah TCM database, and finally to evaluate the usability of the database designed using meCUE. The methodology used was 4D Appreciative Inquiry Model, which included discovery, dream, design, and destiny phases. The advantage of this model was to take a positive core to make any changes instead of finding the weaknesses of the project. Hopefully through the developed database, local Sabahan can take the advantage in identifying the proper usage of existing herbs in their surroundings.
\end{abstract}

Copyright $@ 2018$ Institute of Advanced Engineering and Science. All rights reserved.

\section{Corresponding Author:}

Aslina Baharum,

Faculty of Computing and Informatics,

Universiti Malaysia Sabah,

88400 Kota Kinabalu, Sabah, Malaysia.

Email: aslina@ums.edu.my

\section{INTRODUCTION}

This is a project that further enhances the idea of having a Sabah Traditional Medicine Database (SabahTMed) that was developed in 2016-2017 [1]. Beyond that, based on the observation, similar situation that was faced by the Sabah Traditional Chinese Medicine (TCM) was the limited database designed specifically for seeking about the type or name of TCM and learning the way to treat the disease using the TCM. Most of the basic ideas of the public regarding the TCM are that they are very expensive and difficult to find, hence they tend to seek the modern medical treatment such as pills. However, bear in mind that an abundance of traditional Chinese herbs are exist in our surrounding, and yet many people thought that those are just useless plants or grasses. It is a waste of resources for the Sabahan just because they do not know 
about the TCM. A lot of testimonies show the advantages of TCM compared to modern medicine, for example the index of blood sugar of a diabetic patient was lowered down after the patient consumed the correct TCM for a period, whereas the pills for diabetes are mainly functioned for controlling the blood sugar only. Therefore, it is hoped that the proposed database would help the public to explore the TCM in Sabah. The three main objectives of the project are to identify the validity of the information of the Sabah TCM using mixed method, to develop the Sabah TCM and to evaluate the usability of the database designed using meCUE. The sense of presence of the TCM is too low among the people. That is the reason why the public tends to go to the hospital for treatment. However, as mentioned earlier, those medicines are mainly for controlling instead of treating the illnesses. Besides, the ways of controlling generally vary in different individuals. It is true that, some of them can restore their health, but at the same time, they will need to continuously take more pills in order to control the diseases. Hence, it is hoped that people can notice about the TCM and learn the information of herbs, as an alternative way for them to cure their illnesses.

\section{RELATED WORK}

TCM has formed a unique system to diagnose and cure illnesses [2]. The TCM approach is fundamentally different from that of Western medicine, including the understanding of a human body. The understanding in TCM is based on the holistic understanding of the universe, Daoism. There are a lot of theories involved in the TCM such as, qi, yinyang, five elements, symptoms, and etc. while the treatment is based on the diagnosis and differentiation of syndromes. Although the TCM seems to be useful, there is a lack of formal information that can be used as the main reference. At the same time, most people will prefer to Modern medicine to Chinese Medicine. As for the Chinese Medicine industry in Malaysia, they are facing the problem of prohibition in importing some of the medicines. Hence, it is shocking to know if there are local Traditional Chinese Physicians that use the banned herbal remedies in their treatments. Some people will probably assume that Chinese Medicine means medicines that are imported from China. It is undeniable that there are a lot of Chinese Medicines that are imported from Hong Kong and China. This is because different environments affect the growth of different medicinal plants/bugs. The most obvious example is Cordycepssinensis. Its common name in Chinese can be translated as "winter worm, summer plant". In fact, it is a fungal. In the winter, it will invade the larvae of a moth in the soil, grow inside it, and occupy it, whereas the larvae will move from deep of the soil to the surface of the soil. While in the summer, the fungal will grow upward from the head of the larvae towards the sky like a plant [3]. It is a very nourishing herb and at the same time, it is also valuable. However, there are also herbs that do not exist in the four season country. Hence, the local Sabah TCM is worth to explore.

\subsection{Chinese Herbal Medicine Database}

Chinese Herbal Medicine Database [4] is a web-based platform for The Hong Kong Polytechnics University. The only way for site display of the whole list is by emptying the field and initiating the searching. After a medicine is selected, its medicinal group, source, nature and flavours, meridian affinity, actions, family, part used, indications, cautions, report on adverse effect, and reference are displayed. In addition, the page can be displayed in both English and Chinese languages. The advantage for this site is that the user can compare selected medicine. The categories that can be compared are medicinal group, source, nature and flavours, meridian affinity, actions, family, part used, indications, cautions, report on adverse effect, and reference. While the disadvantage is that the data are still not complete. Some of the details have no data while some do not have the images.

\subsection{Natural Matter and Chinese Medicine Database}

Natural Matter and Chinese Medicine Database [5] is a web-based platform that developed by Taiwan. Hence, most of the contents in this site are in Chinese. The site provides three types of database which are natural matter, Chinese medicine, and prescription. The list of data changes based on selection. After selecting the detail of the medicine, it displays its Chinese name, scientific name in Chinese and Latin, its common name, extraction solvent, used part, solubility, traditional use, storage conditions, indicator ingredient content, picture, details provider, and reference. The search engine used in this site is connected to the Google search engine, "search keywords + site:techtransfer.tmu.edu.tw". The data in this site are not complete since some data are shown as unknown. Besides, it is only written in Chinese, which is quite inconvenient since not everybody knows Chinese.

\subsection{Chinese Medicinal Material Images Database and Medicinal Plant Images Database}

Both Chinese Medicinal Material Images Database [6] and Medicinal Plant Images Database [7] are web-based libraries for Hong Kong Baptist University. The differences between these two sites are Medicinal 
Material used for the material while the Medicinal Plant used for the plant. Medicinal material contains the herb list, its Chinese character and pinyin, Latin and English Name, category and picture for the herb. Besides, it also displays a large picture with the characteristic of the herb, providing the origin and production regions, macroscopic features, quality requirements, properties, functions, processed form, and a link to its. Meanwhile in the medicinal plant consists of the Latin and English name, family and Genus, description, distribution, part used, harvest and processing, chemistry, pharmacology, properties and actions, indications and usage, example, and a link back to medicinal material. Both sites can be displayed in Chinese or English. The advantage is that the data are very detail. Medicinal material has the advantage of having the materials listed according to the physical characteristics category. Besides, both sites are enable user to search data using Chinese, English or Latin keywords of a disease or medicine. However, for the English and Latin keywords, a complete word is needed, such as "Camellia", where it returns nothing if the user type "came" only.

\section{4. zhong yi bai ke: zhong yao}

The zhongyibaike: zhongyao [8] is a mobile-based application. If the name is translated, it means the 'Chinese Medical Encyclopaedia: Chinese Medicine'. Upon opening the application, a list of medicines will appear and it is easy to understand about its function such as digestion medicine. After one of the medicines is selected, a list of herbs to make the medicine will be displayed. If a herb is selected, it displays the source, category, common name in Chinese, scientific name in Latin, flavour, functions, characteristics of the herb, main therapy, processed form, indications, usage, additional note, and literature review of the herb. A picture of the herb will be shown, if available. When offline, this application can still be used, with only some of the features cannot be used. In terms of its search engine, it can only be done by using the keyword or the name of the herb. The only disadvantage of the apps is that it is written in Chinese.

\section{5. zhong yao jian kang bai ke}

The zhongyaojiankangbaike [9] is a mobile-based application. The English translated name for the application is 'Healthy Chinese Medicine Encyclopaedia'. After opening the apps, it will display some of the medicines. By selecting the category of the herbs, it will display a list of TCM. If one of the herbs is selected, it will display its source, name in Chinese character with pinyin, and English if it is available in its database. Besides, it also gives the function and valuable usage, effective, and clinical application to the user. In addition, it has a share feature, also other features such as combinable medicine, Chinese medical, and health features. Meanwhile in terms of the searching activity, when using keywords, it will display all information connected to the keyword, which is quite confusing to the user. Besides, the details of the herbs are displayed and explained in paragraphs, which is too lengthy and tedious for the user to identify specific information. In addition, this application can only be used when the user is online, and it is totally in Chinese version.

\section{6. zhongchun tang zhongyao cha xun}

The zhongchun tang zhongyao cha xun [10] is a mobile-based application that is hosted by Zhong Chun Tang, a Chinese Medical Store in Taiwan. The translation of the application is 'Chinese Medicine Inquiry of Zhong Chun Tang'. Upon opening the application, it displays three main features; inquire TCM, inquire the combination medicine, and about us. When the TCM is selected, a list will be displayed, together with a picture, common name, English name, flavour, main function, and explanation of the function. Meanwhile in terms of the combination of medicine, another list will be displayed, as well as how to make it and its functions. In addition, it also provides suggestions of other TCM for someone with different symptoms. The user can select the TCM and see the details as in the TCM list. Besides, the application can be used while offline. As for the search function, it will display all results that consist the keywords. For the language, it is totally in Chinese version.

\section{METHODOLOGY}

The overall methodology used for the whole project was the 4D appreciative inquiry model. Appreciative inquiry (AI) is a philosophy and methodology for positive change. Based on simple assumption on human systems, it is founded by moved in the direction such as the fields of study, the things that focus on, and the ways of communication [11]. The AI process generally follows the AI 4-D Process which was used in this project. There were four processes which were discovery, dream, design, and destiny can see in Figure 1. In short, AI suggested human organizing and change, which also focused on realizing, and finally appreciation of what worked well. 


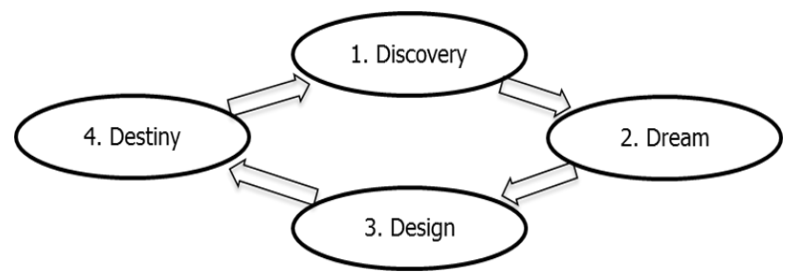

Figure 1. Appreciative inquiry 4-D process

Figure 2 shows the adapt process from Figure 1; In the first D; Discovery, the efforts focused on elaborating the positives and strengths to look for the best. In other words, it was to identify and appreciate what would work. In the second D; Dream, the time was spent to contemplate what and how it might be. In simple sentence, it was to imagine what it might be. In the third D; Design, the steps involved turning speculation into reality. This phase means the development of the system, while its structures were leveraging the best of what was and what had been investigated in previous phases. In the final D; Destiny, the time was spent to move towards the progress and realities of the desired state. In this phase, the proposed design was implemented or delivered.

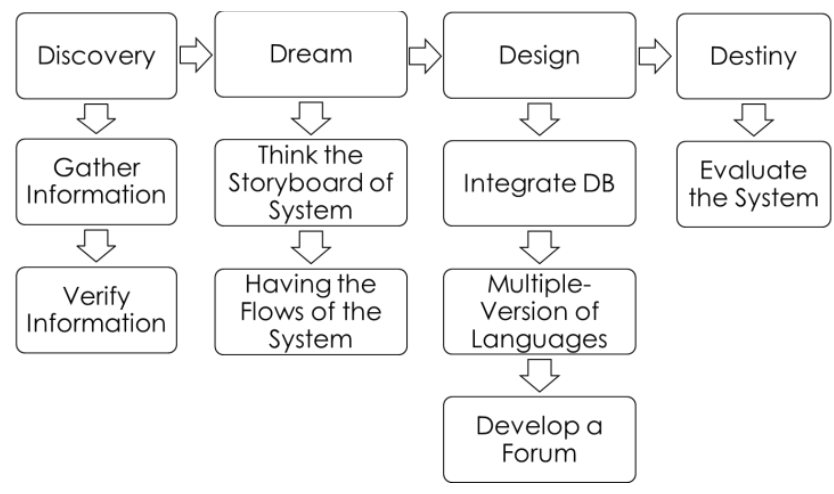

Figure 2. Appreciative inquiry 4-D process for SabahTCM

Discovery phase in this project was the planning on the project. Data collection and verification were done in this phase. The analysis of existing software was also done to complement the database. Qualitative approach is primarily exploratory research which is used to uncover the trends in thoughts and opinions which dive deeper into the problems [12]. Meanwhile the quantitative approach adopts experimental and computational method. Here, the data of TCM were collected from multiple sources. The sources of the data were the online reference, articles in the magazine, and the printed version of manual books that are published by various organizations. After collecting the data, all the data were referred to the expert, either the Herbalist, Traditional Chinese Pharmacist, Traditional Chinese Physician, or those who have experiences and skills in using the TCM. The way to validate the data was through questionnaires and interviews. For the dream phase, the flows of the system were the outcome. The storyboard and all the diagrams were done here. For the design phase in this project, the user interface showed the results, the data was integrated into the database, while multiple version of languages was added into the system. In addition, a forum was developed. For the destiny phase in this project, the evaluation was taken. The methodology of the evaluation was using the meCUE questionnaire which was to evaluate the key aspects of the user's experience.

\section{RESULTS AND ANALYSIS}

\subsection{Data Collection}

The data of TCM were gathered from the online and printed references. Since the sources that were used for the data collection were valid sources, the rest was just to validate whether the herb is a Chinese medicine or not. The data gathered for validation included the vernacular name, others name, scientific name and Chinese name of the herb. For example, Carpet Weed is the vernacular name, while its other names are 
'PokokPecahKaca' and 'PokokPecahBeling'. The scientific name is Strobilanthescrispa Bl., and lastly the Chinese name are po shi cao, heimianjiangjun and cui ye ma lan. The main printed references used were Medicinal Plants in Sabah: Volume 1 [13] and Medicinal Plants in Sabah: Volume 2 [14]. These series of books are the manuals on medicinal plants of Sabah, which were published by Forest Research Centre of Sabah Forestry Department. The authors have been studying about the medicinal plants for about three decades, while one of the authors has been practicing traditional Chinese medicine for many years. Hence, these series are biased towards Chinese herbal medicine. The information included inside these series of manuals are the group, family, scientific name, vernacular name, habit and occurrence, taste, energy and characteristics, function, uses, parts used, folk prescription, and the family and vernacular names in Chinese of the herbs.

There are a lot of websites related to the TCM nowadays. The websites include formal references that are published by universities or organisations and informal references posted by the public. Although there are a lot of formal online references, most of the herbs in the printed references are not found in those formal online references. This is because most of the formal online references are published by those institutes in the People's Republic of China, Hong Kong and Taiwan, hence the data of TCM in those references are from China. The failure in matching the data showed that the herbs between China and Malaysia are significantly different. The main reason of the differences is due to the habits and occurrences of the herbs. Hence the different climates; China's four season climate and Malaysia's equatorial climate, have a huge impact on the growth of the herbs. For example, the Traditional Chinese Herbal Plants of Globinmed website [15] was developed in China. On the website, there are 147 data, but only five of the herbs can be found in the manuals of Medicinal Plants in Sabah: Volume 1 and Medicinal Plants in Sabah: Volume 2. In other words, the online references from overseas cannot be used as the sources for data collection. Hence, the sources of online references were narrowed down and aimed for the local, which is Malaysia, especially in Sabah.

\subsection{Results}

After gathering and comparing those data, about 150 data were collected. The data included the herbs' local name in English and Malay if available, as well as the scientific name. Besides that, the families of the herbs were also recorded. In addition, the parts of the herbs to use, the expertise functions of the herb, and the diseases that the herbs may treat were also gathered. The database can be access using the url: https://sabahtcm.000webhostapp.com.

\section{DEVELOPMENT OF SABAH TCM}

SabahTCM allows the users to search for the information of the TCM through the name of the herbs and the type of diseases that the herb treats. Besides, a forum was implemented to let the users and the admin to exchange their opinions regarding the TCM. Finally, considering the different language background of the users, both English and Chinese versions were implemented in the SabahTCM as an option for them to understand the information. There are three main modules; Herbs, Forum and Bilingual Module. Herbs Module - In this module, users may look through the information of the herbs. Besides that, they can also search the herbs through the keywords, which are the name of herb and the disease that the herb treats. Forum Module - consists of two categories, which are Question category as well as Opinion and Suggestion Category. Users can add topics and respond to any comments OR respond and give comments. However, the topic and comment will only show in the forum after being reviewed by the admin to filter up the spam. Besides that, a topic search engine is also provided. Users can fill the keyword of a topic to search about the topic. Bilingual Module - SabahTCM is proposed to have an alternative approach to the users from different background of languages to understand the information of SabahTCM. Hence, English and Chinese versions are offered on the site. However, for the forum module, bilingual topics and comments from the users or admin are prohibited. This is to prevent any misinterpretation and misunderstanding of ideas that a person expresses. Users can switch between languages through the link on the upper right hand side. Figure 3 shown interfaces of Sabah TCM. 


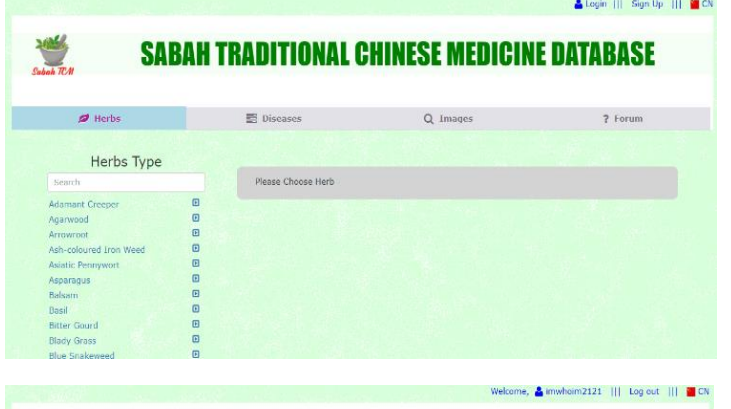

\section{SABAH TRADITIONAL GHINESE MEDIGINE DATABASE}

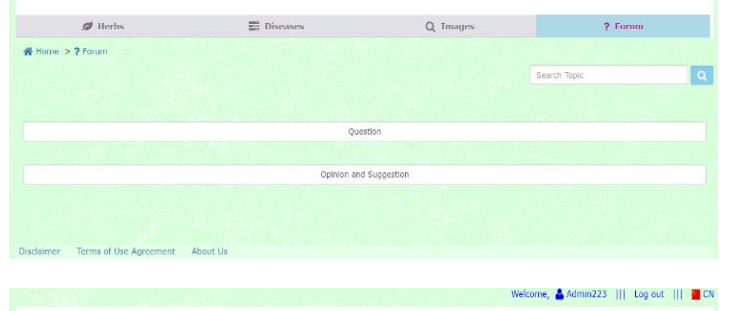

\section{SABAH TRADITIONAL CHINESE MEDIGINE DATABASE}

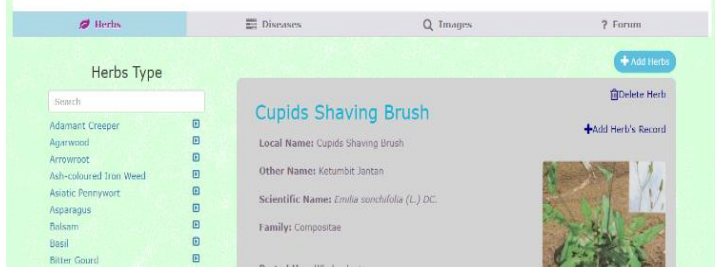

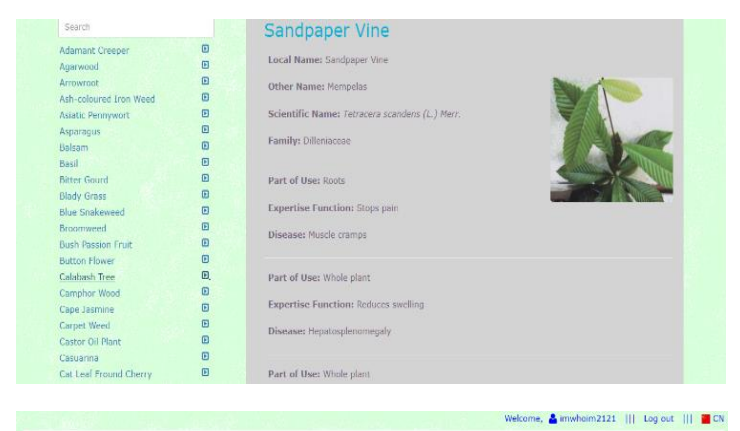
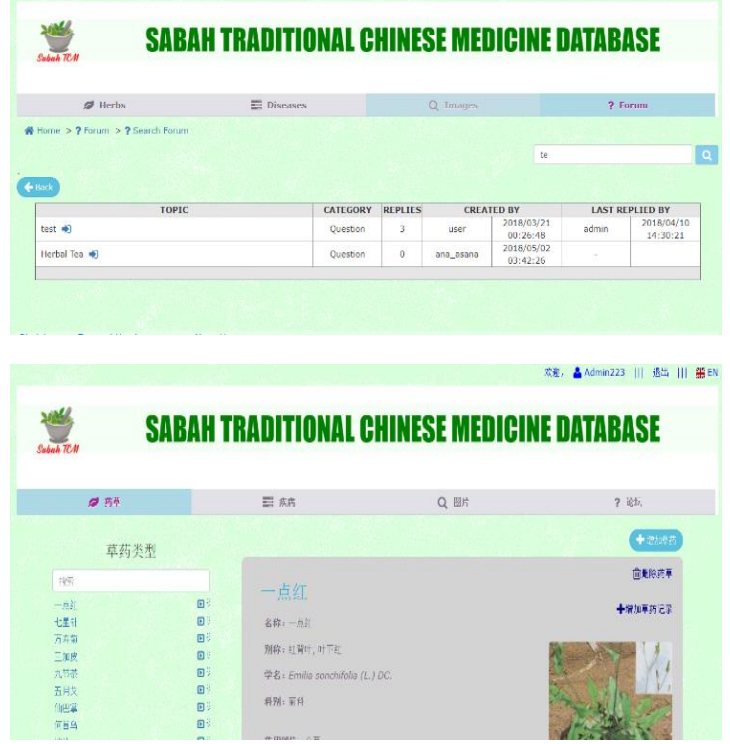

Figure 3. Interfaces of Sabah TCM

\section{USABILITY TESTING}

The usability testing was based on the evaluating of system using meCUE questionnaire. In meCUE, it had four different modules which included the perception of characteristics, the users' emotions, with the consequences, and the overall assessment toward the system. The questionnaire was adapted and modified from the original meCUE questionnaire. Next, it was set in an online Google form. The distribution of questionnaire was done face-to-face to the local Sabahan. The number of respondents of this usability testing was 30 people. The evaluation of this testing was according to the modules mentioned above. The first module was the users' perception of instrumental qualities of the system by the characteristics of the usefulness and usability. The second module was the users' perception of non-instrumental qualities of the system through visual aesthetics, status and commitment of the users towards the system. The third module was the users' emotions, in both positive and negative emotions. The forth module was the consequences of use by the users towards the system that evaluated the intension of use and the product loyalty. Finally, the last module was the overall evaluation about the system by the users. The first four modules had their respectively set of statements while each statement was assigned to a numeric value for evaluation from strongly disagree with the value of 1 until strongly agree with the value of 7 . Meanwhile the last module had a range value from -5 to 5 . The Figure 4-8 display the results of the evaluation in mean values. 


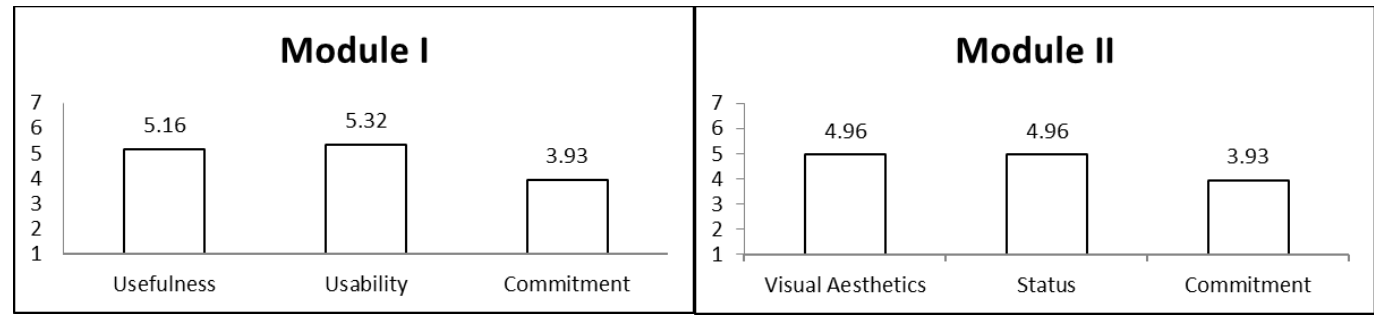

Figure 4. Module I perception of instrumental qualities
Figure 5. Module II perception of noninstrumental qualities

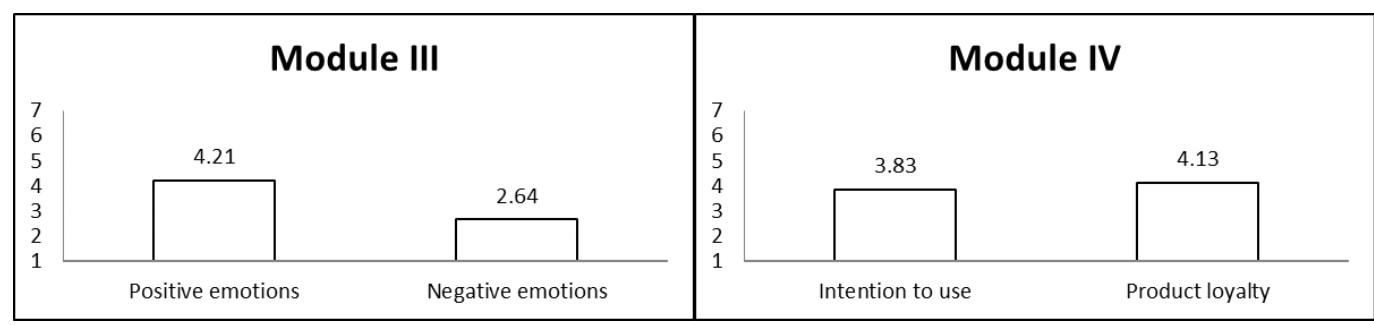

Figure 6. Module III user emotions

Figure 7. Module IV consequences of use

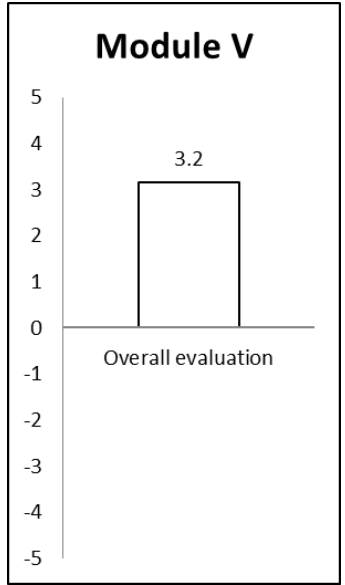

Figure 8. Module V overall evaluation

\section{DISCUSSION}

Through the usability testing, the function of Sabah TCM can be said as a useful with usability characteristics. Meanwhile the interface of Sabah TCM can be considered as having an interesting design and the respondents felt that their understanding was enhanced when using Sabah TCM. In terms of the commitment of responses towards Sabah TCM, their feelings were neutral or the same, whether they were using Sabah TCM or not. As for the emotions, the responses gave response that Sabah TCM would not let them have too much positive emotion, and will not let them felt negatively. Besides, they also responded that, they would not be eager to use Sabah TCM except when they needed to check or search the information regarding the traditional Chinese medicine. As for the product loyalty, they said that they might continue to use Sabah TCM since it was difficult to find online references regarding the local traditional Chinese medicine. Finally as the overall evaluation of Sabah TCM, they had a great experience using Sabah TCM.

\section{CONCLUSION}

The main concern for this project was to develop a system according to the idea of an existing system, SabahTMed. The next idea was to develop a similar database system that consisted of the 
information of TCM in Sabah. As a result, Sabah TCM Database system was developed. The purpose of Sabah TCM was to store all the information regarding to traditional Chinese medicine that can be found in Sabah. The data gathered for the system would be very useful for the users in the future. Through the Sabah TCM system, users can get the herbs' information through the name of the herbs and the diseases that are treated. Due to the decrease number of Traditional Chinese Pharmacists and Traditional Chinese Physicians over the years, the price for Traditional Chinese Medical consultation is getting higher. This project provided the information that has already being verified and validated by expertise, hence the users may refer to the data for self-consultation, before they seek for Herbalist or Chinese Physicians.

In the future, the maintenance of Sabah TCM requires more valid and verified data for the completeness of the dataset in the database. The data in the current project looks quite a lot, but as there are other herbs that can be found in Sabah, a lot of data are still not listed in the database. Besides, there are still other characteristics and information of the medicines that can be included in the data. Meanwhile for the forum, it would be useful to have a pin function in the future, so that the admin can pin any announcements or important rules to prevent any spam messages posted by the users. In addition, any post by the users need to be reviewed by the admin before it appears in the forum. Hence, an additional function that allows the users to edit their posts is needed.

\section{REFERENCES}

[1] B. Aslina, et al., "Sabah Traditional Medicine Database and Application: SabahTMed," Advanced Science Letters, vol/issue: 24(3), pp. 1834-1838, 2018.

[2] Traditional Chinese Medicine, "Introduction to Chinese Medicine/TCM," 2009. Retrieved from Traditional Chinese Medicine Information Page: http://www.tcmpage.com/.

[3] F. Mo, "Mei Tian Yi Wei Cao YaoL Dong Chong Xia Cao, Dong Chong Xia Cao, Rang WoShuo Ni Shen Me Hao?" 2016. Retrieved from Cai Fu: https://m.91ddcc.com/t/105514.

[4] The Hong Kong Polytechnic University, "Chinese Herbal Medicine Database," 2015. Retrieved from Chinese Herbal Medicine Database: http://herbaltcm.sn.polyu.edu.hk/.

[5] Tai Bei Yi Xue Da XueJiShu Jiao Yi Ping Tai, “Tian Ran Wu Yu Zhong Yao Zi Liao Ku,” 2017. Retrieved from Tai Bei Yi Xue Da XueJiShu Jiao Yi Ping Tai: http://techtransfer.tmu.edu.tw/medicine/medicine1.php.

[6] Hong Kong Baptist University, "Chinese Medicinal Material Images Database,” 2012. Retrieved from Hong Kong Baptist Library: http://library.hkbu.edu.hk/electronic/libdbs/mmd/index.html.

[7] Hong Kong Baptist University, "Medicinal Plant Images Database," 2007. Retrieved from Hong Kong Baptist University Library: http://library.hkbu.edu.hk/electronic/libdbs/mpd/index.html.

[8] Impression Mobile, “Zhong Yi BaiKe: Zhong Yao,” Eglinton Ave West, Toronto, Canada, 2017. Retrieved from https://play.google.com/store/apps/details?id=fmo.tcmmedicinech.

[9] Ken, "China," 2016. https://play.google.com/store/apps/details?id=org.zywx.wbpalmstar.widgetone.uex11118708.

[10] Mocnac, "Zhong Chun Tang - Zhong Yao Cha Xun," China, 2016. Retrieved from https://www.google.com/url?sa=t\&rct=j\&q=\&esrc=s\&source=web\&cd=1\&ved=0ahUKEwiulI2DhonYAhVGLo8 KHYZXBBMQFggoMAA\&url=https\%3A\%2F\%2Fplay.google.com\%2Fstore\%2Fapps\%2Fdetails\%3Fid\%3Dcom .alemocni.cml.zhongchuntang\&usg=AOvVaw3UKG7q4IGglTg_g9pN0n-W.

[11] Corporation for Positive Change, "Appreciative Inquiry (AI)," 2015. Retrieved from Corporation for Positive Change: https://positivechange.org/how-we-work/appreciative-inquiry-ai/.

[12] S. Wyse, "Difference between qualitative and quantitative research?" 2011. Retrieved from SnapSurveys: https://www.snapsurveys.com/blog/qualitative-vs-quantitative-research./

[13] Y. Lee, et al., "Medicinal Plants in Sabah," Sandakan, Sabah, Malaysia: Sabah Forestry Department, vol. 1, 2015.

[14] Y. Lee, et al., "Medicinal Plants in Sabah," Sandakan, Sabah, Malaysia: Sabah Forestry Department, vol. 2, 2016.

[15] Global Information Hub on Integrated Medicine, "Traditional Chinese Herbal Plants - Professional Data," 2015. Gloinmed:http://www.globinmed.com/index.php?option=com_content\&view=category\&id=22\&Itemid=154. 